ISSN 2079-9292

www.mdpi.com/journal/electronics

Review

\title{
Emerging Transparent Conducting Electrodes for Organic Light Emitting Diodes
}

\author{
Tze-Bin Song ${ }^{1,2}$ and $\mathrm{Ning} \mathrm{Li}^{2, *}$
}

1 Department of Materials Science \& Engineering, University of California, Los Angeles, CA 90095, USA; E-Mail: tzesong@gmail.com

2 IBM T. J. Watson Research Center, 1101 Kitchawan Road, Yorktown Heights, NY 10598, USA

* Author to whom correspondence should be addressed; E-Mail: lini@us.ibm.com; Tel.: +1-914-945-1689.

Received: 21 January 2014; in revised form: 1 March 2014 / Accepted: 12 March 2014 /

Published: 21 March 2014

\begin{abstract}
Organic light emitting diodes (OLEDs) have attracted much attention in recent years as next generation lighting and displays, due to their many advantages, including superb performance, mechanical flexibility, ease of fabrication, chemical versatility, etc. In order to fully realize the highly flexible features, reduce the cost and further improve the performance of OLED devices, replacing the conventional indium tin oxide with better alternative transparent conducting electrodes (TCEs) is a crucial step. In this review, we focus on the emerging alternative TCE materials for OLED applications, including carbon nanotubes (CNTs), metallic nanowires, conductive polymers and graphene. These materials are selected, because they have been applied as transparent electrodes for OLED devices and achieved reasonably good performance or even higher device performance than that of indium tin oxide (ITO) glass. Various electrode modification techniques and their effects on the device performance are presented. The effects of new TCEs on light extraction, device performance and reliability are discussed. Highly flexible, stretchable and efficient OLED devices are achieved based on these alternative TCEs. These results are summarized for each material. The advantages and current challenges of these TCE materials are also identified.
\end{abstract}

Keywords: transparent electrode; organic light emitting diode; carbon nanotube; metallic nanowire; graphene; conductive polymer 


\section{Introduction}

Organic light emitting diode (OLED) has emerged as a potential candidate for next generation flexible, large-area, high resolution display and solid state lighting panels, because of its high color quality, attractive appearance, ease of fabrication, low manufacturing and materials cost, etc [1]. With great efforts from both academia and industry, the OLED has been developed based on small molecule and polymer materials and also fabricated with both vacuum deposition and solution processes [2-5]. In the past few decades, researchers have been focusing on improving the device efficiency and lowering the manufacturing cost. Today, OLED displays are becoming dominant in the high-end market. OLED lighting is also on the verge of becoming widely commercially available, and its performance is competitive with that of its inorganic counterparts.

The basic OLED structure is composed of a stack of several layers: anode/hole transport layer (HTL)/emission layer (EL)/electron transport layer (ETL)/cathode, as shown in Figure 1a. [6] The first OLED was developed by Tang and VanSlyke in 1987 with the structure of an indium tin oxide (ITO)/aromatic diamine/8-hydroxyquinoline aluminum $\left(\mathrm{Alq}_{3}\right) / \mathrm{Mg}$ - $\mathrm{Al}$ metal electrode [7]. Since then, ITO glass has been commonly used as the anode for OLEDs, because ITO simultaneously provides good transparency and conductivity [8]. Moreover, the work function of ITO is around $4.7 \mathrm{eV}$, which forms a low barrier for hole injection into the emission layer made of commonly used organic materials (Figure 1b) [9]. Despite these advantages, ITO is far from being a perfect candidate for OLED applications for the following reasons. First, it is not ideal for highly flexible electronics, due to its brittleness. Under mechanical bending or stretching, crack generation in the ITO film leads to much deteriorated electrical properties [10]. Second, the sputtering deposition of high quality ITO is a low throughput process and requires elevated temperature. Solution processed ITO also requires high temperature annealing to achieve a good conductivity [11]. It is vital therefore to only use substrates that are stable at high temperatures, which means an increased substrate cost and much reduced performance on plastic substrates. Furthermore, due to the widespread application of ITO as the transparent conducting electrode (TCE) for various optical devices and the limited global reserve of indium, the price of ITO will rise dramatically and further raise the cost of OLEDs. In addition, ITO does not offer ideal performance for OLEDs. It has significant light reflection and also traps the light in the waveguide mode. Its conductivity needs to be further improved, as well, for large area devices.

Considering all these factors, there has been increasing interest and an urgent need to look for alternative TCE materials to replace the conventional ITO. These TCE materials should be highly conductive and optically transparent; meanwhile, they should also be low cost and enable new attractive features. Here, we review the recent progress on the promising next generation TCEs. We focus our attention on the following materials: carbon nanotubes (CNTs), metallic nanowires, conducting polymers and graphene. These materials have shown the potential to fulfill standard requirements on the sheet resistance and transmission values of TCE and can be formed by low-cost processes, such as spin coating, spray coating and even roll-to-roll processes [12]. The sheet resistance and transmittance of these materials is summarized in Figure 1c using the solar spectrum as a reference for transmission evaluation. Moreover, OLED devices demonstrated with these techniques show great potential for future highly flexible, foldable and wearable opto-electronics. We summarize the progress 
of each of these TCE materials with the device performance achieved and give comparisons between these techniques.

Figure 1. (a) Schematic diagram of the organic light emitting diode (OLED) structure; (b) engery level diagram of a simple OLED device consisting of $N, N^{\prime}$-Bis(3methylphenyl)- $N, N^{\prime}$-diphenylbenzidine (TPD), $N, N^{\prime}$-Di(1-naphthyl)- $N, N^{\prime}$-diphenyl-(1,1'biphenyl)-4,4'-diamine (NPB), 4,4'-Bis( $N$-carbazolyl)-1,1'-biphenyl (CBP), Bathocuproine (BCP), and Tris-(8-hydroxyquinoline)aluminum (Alq3); (c) sheet resistance and transmission chart for various types of transparent conducting electrode (TCE) materials including carbon nanotube (CNT), silver nanowire (AgNW), conductive polymers, and graphene.

a)

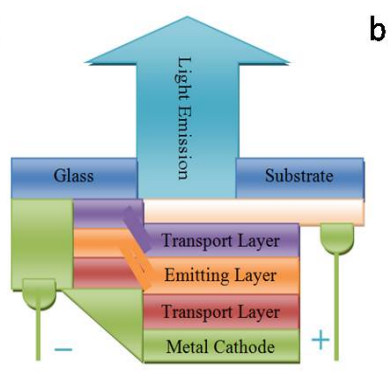

b)

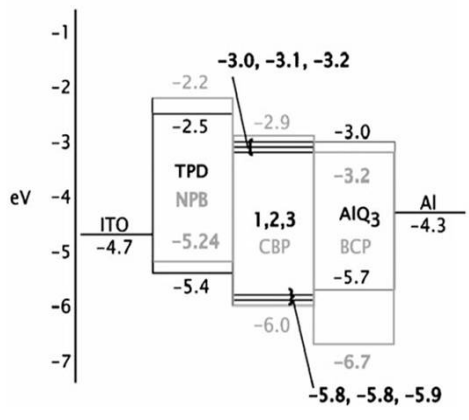

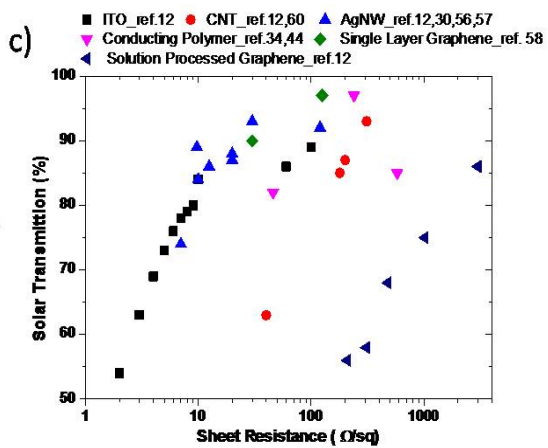

\section{Carbon Nanotubes}

The carbon nanotube (CNT) network is the first nanostructured TCE investigated for OLEDs, leading to a boom of interest in this decade [13]. CNTs exhibit a unique electrical property in that they can be both metallic and semiconducting [14]. Because of this, they are widely applied as high-performance flexible transparent transistors, optical modulators, flexible emitters, as well as TCEs [15-17]. Metallic CNTs have a suitable work function (4.7-5.2 eV) for the application as anodes in OLEDs $[18,19]$. In addition, the high stability, flexibility and mobility of CNTs make the CNT network a potential candidate to replace the rigid ITO substrate, while avoiding the contamination of the organic layers from the oxygen atoms in ITO.

Zhang et al. first developed large area CNT sheets (meter long, $5 \mathrm{~cm}$-wide) as the electrode for OLED, and this nanotube sheet was reported to be as strong as the steel (Figure 2a) [13]. This report demonstrated the huge potential of CNT networks' application in optical electronics and opened a new direction for the nanostructured TCE. Zhang et al. tested various CNTs from different growth methods [20]. The arc discharge nanotubes showed better performance compared to high-pressure $\mathrm{CO}$ conversion (HiPCO) nanotubes in surface roughness, sheet resistance and transparency. A sheet resistance of $\sim 160 \mathrm{ohm} / \mathrm{sq}$ at $87 \%$ transparency can be achieved when the CNTs network is treated with $\mathrm{SOCl}_{2}$, as shown in Figure 2b,c. Li et al. demonstrated that a poly(3,4-ethylenedioxythiophene) poly(styrenesulfonate) (PEDOT:PSS) layer could play an important role in planarizing the roughness from CNT networks (Figure 2d) and decreasing the hole injection barrier from the CNTs to a polymer blend hole transporting layer poly(9,9-dioctylfluorene-co- $N$-(4-butylphenyl)diphenylamine) (TFB)+ 4,4'-bis[( -trichlorosilylpropylphenyl)phenylamino]biphenyl (TPD-Si 2 ), which could reduce the 
leakage current [21]. With a PEDOT:PSS layer modified with methanol, a surface roughness less than $1 \mathrm{~nm}$ can be achieved, due to better PEDOT:PSS wetting onto the CNT network.

Ou et al. further modified the surface of the carbon nanotube network with PEDOT:PSS composite $\left(\mathrm{PS}^{\mathrm{C}}\right.$ ) coating, which contained polyethylene glycol (PEG) additive in Baytron P500 and used $\mathrm{HNO}_{3}$ acid treatment to improve the conductivity of the CNT network and the band alignment for hole injection [22]. Outstanding performance with a maximum luminance of $\sim 9000 \mathrm{~cd} / \mathrm{m}^{2}$ and a luminance efficiency (LE) of $\sim 10 \mathrm{~cd} / \mathrm{A}$ at $1000 \mathrm{~cd} / \mathrm{m}^{2}$ was achieved, which was comparable to devices on ITO substrates. Yu et al. explored the capability of CNT transparent electrodes as the cathode and anode for flexible and transparent organic light emitting diodes by a lamination method [23]. Furthermore, stretchable OLEDs based on a CNT network as the TCE was built. The electroluminescent efficiency of the devices can be sustained under a $45 \%$ strain, which cannot be achieved for traditional ITO substrates [24]. The device stability with the CNT electrode exhibited comparable lifetime with that of the ITO electrode, and the acid resistivity of the CNT electrode to PEDOT:PSS is better than that of ITO electrode for long-term operation.

Figure 2. (a) Photograph of an OLED that uses an multi-wall nanotube (MWNT) sheet as the anode and poly(3,4-ethylenedioxythiophene) poly(styrenesulfonate) (PEDOT:PSS)/ Poly[2-methoxy-5-(2-ethylhexyloxy)-1,4-phenylenevinylene] (MEH-PPV) /Ca/Al [13]; (b) patterned multilayer single wall CNT/PEDOT:PSS/NPB/Alq $3 / \mathrm{LiF} / \mathrm{Al}$ [20]; (c) device performance: photoluminescence spectrum, current density $v s$. voltage bias curve, brightness vs. voltage bias and quantum efficiency as a function of current density [20]; (d) surface roughness of PEDOT:PSS $\sim 4 \mathrm{~nm}$ and methanol-modified PEDOT:PSS $\sim 0.96 \mathrm{~nm}$ on a CNT network [21]; (e) the luminescence vs. the voltage of an OLED with PEDOT:PSS composite $\left(\mathrm{PS}^{\mathrm{C}}\right)$-modified CNT on Polyethylene terephthalate (PET) substrate [22].

a)

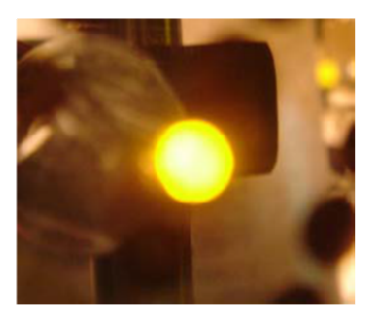

d) b)

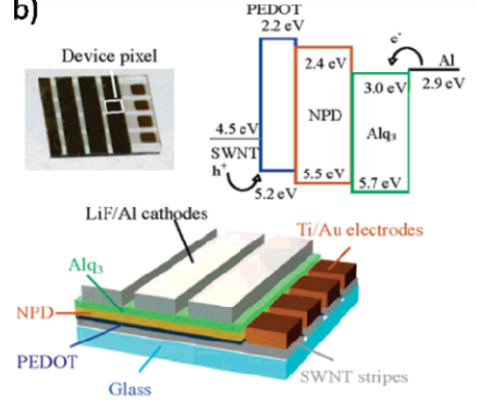

c)
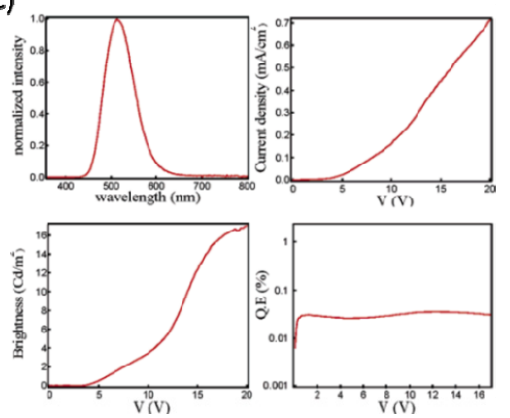

e)

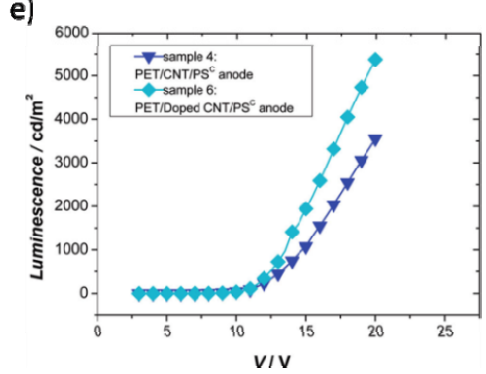

Reproduced with permission from Zhang et al., Science, published by the American Association for the Advancement of Science, 2005 [13]; Zhang et al., Nano Letter, published by the American Chemical Society, 2006 [20]; Li et al., Nano Letter, published by the American Chemical Society, 2006 [21]; and Ou et al., ACS Nano, published by the American Chemistry Society, 2009 [22]. 
Figure 3. (a) Solar photon flux-weighted transmissivity $v$ s. sheet resistance for Ag gratings (blue line), ITO (red dotted line), CNT meshes $(\Delta)$ and Ag nanowire meshes ( $\square$ ) deposited on a glass substrate. The Ag line width is a $40 \mathrm{~nm}$ and a $400 \mathrm{~nm}$ grating period [26]. (b) Normalized radiant intensity, color coordinates vs. viewing angle and photographic image of four operating nanowire (NW)-OLEDs [28]. (c) Photographs of a polymer light-emitting electrochemical cell (PLEC) (original emission area, $5.0 \times 4.5 \mathrm{~mm}^{2}$ ) biased at $14 \mathrm{~V}$ at specified strains [29]. (d) Colors from blue to red can be selected by different period nanowire arrays [32].

a)

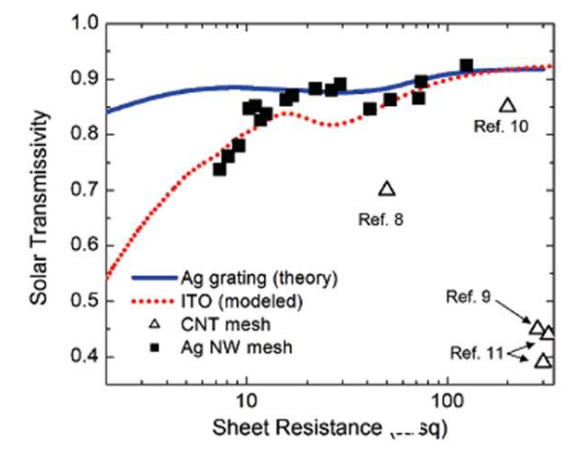

c)

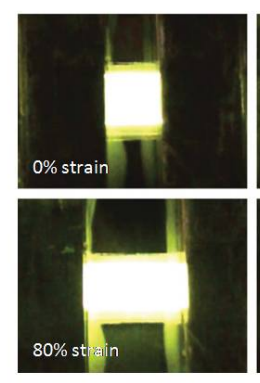

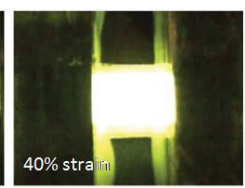

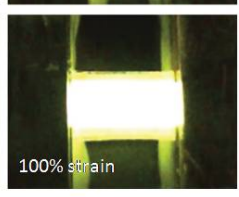

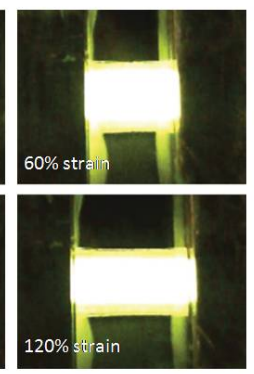

b)

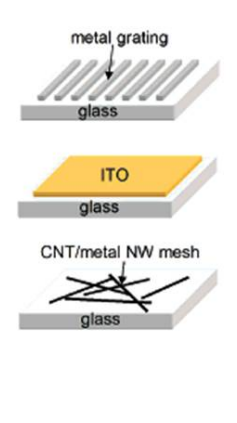

d)
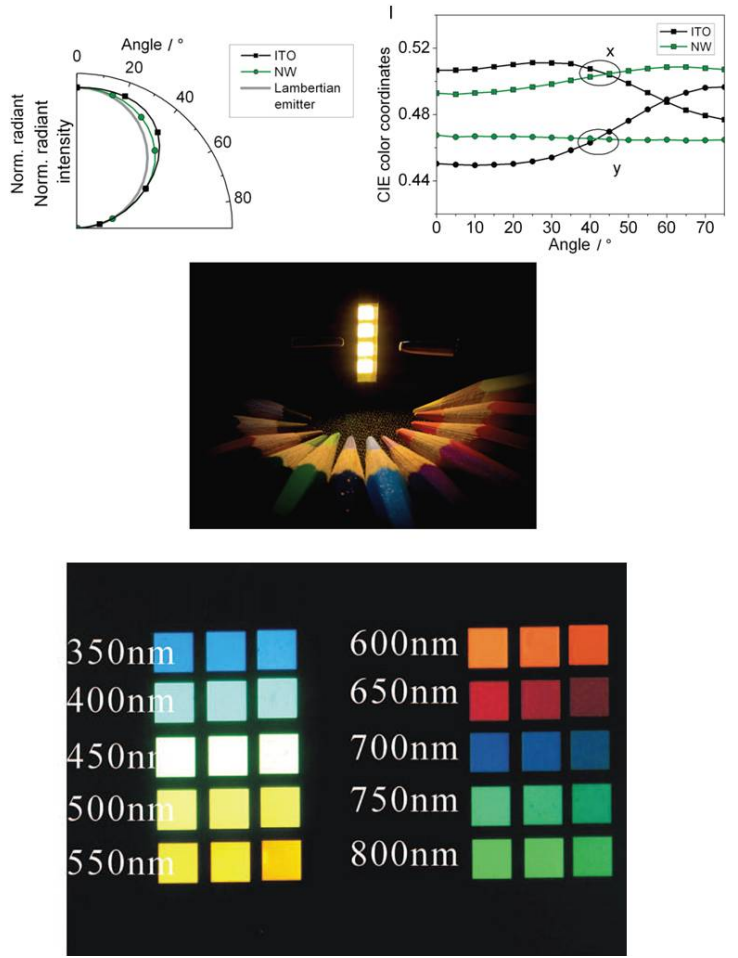

Reproduced with permission from Lee et al., Nano Letter, published by the American Chemical Society, 2008 [26]; Gaynor et al., Advanced Materials, published by John Wiley \& Sons, Inc., 2013 [28]; Liang et al. Nature Photonics, published by Macmillan Publishers Limited, 2013 [29]; and Hsu et al., Apply Physics Letter, published by the American Institute of Physics, 2008 [32].

\section{Metallic Nanowires}

Recent studies on the metallic nanowire's application in optical electronics have attracted a lot of attention. Similar to CNTs, high conductivity from the metal material and high transmittance from the open space between nanowires make the metallic nanowire a potential candidate as the TCE. Compared with CNTs, the metallic nanowire network shows better sheet resistance and transmission values, because the wire to wire contact resistance can be reduced by thermal treatment [25]. The low contact resistance between nanowires can significantly reduce the power loss on the electrodes. However, the metallic nanowire network requires PEDOT:PSS or other hole transport materials to ensure efficient hole injection as an anode, which slightly restricts the fabrication process. Lee et al. demonstrated the potential of a silver nanowire network TCE on a glass substrate with a sheet resistance of $16 \mathrm{ohm} / \mathrm{sq}$ and an average transmittance of $86 \%$ between the wavelengths of 400 and $800 \mathrm{~nm}$, which is comparable to commercial ITO substrates (Figure 3a) [26]. Yu et al. first 
demonstrated a composite electrode in which the silver nanowires were embedded in cross-linkable polyacrylate substrate, which could successfully replace the traditional rigid glass substrate [27]. This result opened up the possibility of realizing the high flexibility and high performance OLEDs by incorporating a solution processed metallic nanowire network.

Gaynor et al. investigated the angular dependence of white OLEDs using silver nanowires embedded in poly(methyl methacrylate) (PMMA) as the electrode [28]. The scattering of the silver nanowire network kept a stabilized viewing angle characteristic with reduced color shift and better Lambertian emission for the OLED. By further incorporation of light outcoupling techniques, a power efficiency of $54 \mathrm{~lm} / \mathrm{W}$ was achieved, as shown in Figure 3b. Liang et al. reported an elastomeric polymer (polyurethane acrylate (PUA)) -based silver nanowire substrate with yellow light-emitting polymers consisting of ethoxylated trimethylolpropane triacrylate (ETPTA), polyethylene oxide (PEO) and lithium trifluoromethanesulphonate (LiTf), and the efficiency was kept at $2.5 \mathrm{~cd} / \mathrm{A}$ under $120 \%$ strain (Figure 3c) [29]. The concern for metallic nanowire electrode is the instability, due to Rayleigh instability and contact ripening, resulting in the loss of the conductive path during operation. These might be the challenges for having long lifetime OLED devices [30,31].

The improvement of the silver nanowire TCE provided a platform for OLEDs to reach wider applications on display and lighting. Furthermore, the dimension of the metallic nanowire could affect the light scattering, light coupling and sheet resistance to transmission values of the TCE, providing us with an additional degree of freedom in improving the device performance. Aligned metal nanowire fabricated by a vacuum process was reported to have improved light outcoupling of the OLEDs (Figure 3d) [32]. The optical effect of the metallic nanowire on OLED and the alignment control of the nanowire through fabrication are still under investigation.

\section{Conductive Polymers}

Among various types of conductive polymers, PEDOT:PSS and polyaniline (PANI) are currently the most popular materials to replace the conventional ITO electrode. These two materials are well-studied, conjugated polymers with excellent mechanical stability, flexibility and, more importantly, they can achieve a high conductivity and transparency.

It was shown that PANI has the potential as a solution-processable TCE by Cao et al. [33]. They discovered that the camphor-sulfonic acid (CSA) doped PANI (PANI:CSA), which is soluble in $\mathrm{m}$-cresol or chloroform, is conductive $(<10 \mathrm{~S} / \mathrm{cm})$ and optically transparent. Gustafsson et al. successfully fabricated the PANI:CSA film on Polyethylene terephthalate (PET) substrate as the anode and demonstrated the first flexible polymer light emitting diode (PLED) device [5]. However, PANI:CSA film needs to be thicker than $250 \mathrm{~nm}$ to achieve $160 \mathrm{ohm} / \mathrm{sq}$, while the transparency is about $70 \%$ under such a thickness (Figure 4a) [34]. Fehse et al. reported a new dispersion of PANI (D1033), which has a conductivity of $200 \mathrm{~S} / \mathrm{cm}$ and a lower absorbance at $750 \mathrm{~nm}$ in the visible region than that of PANI:CSA (Figure 4b) [35]. Even though the improvement of the PANI properties resulted in better device performance, it is still lower than that of the commercial ITO substrate. PEDOT:PSS was invented in 1991 by Bayer [36] as an antistatic coating material. Cao et al. first introduced PEDOT:PSS into the PLED as a TCE with $500 \mathrm{ohm} / \mathrm{sq}$ and $75 \%$ transmission within the visible light region [37]. After that, PEDOT:PSS became commonly used as the hole injection layer for OLEDs, as 
well. In order to improve the properties of the PEDOT:PSS, many treatments have been applied to improve the conductivity without losing the transparency. Kim et al. investigated different organic solvents, including dimethyl sulfoxide (DMSO), $N, N$-dimethyl formamide (DMF) and tetrahydrofuran (THF), and the addition of the high boiling polar solvent DMSO produced the highest conductivity among the three [38]. Ouyang et al. reported using ethylene glycol (EG) as a polar additive and acquired PEDOT:PSS thin films with a conductivity up to $160 \mathrm{~S} / \mathrm{cm}$, demonstrating a comparable performance to ITO/PEDOT:PSS on Poly[2-methoxy-5-(2-ethylhexyloxy)-1,4-phenylenevinylene] (MEH-PPV) PLED devices [39]. Fehse et al. used a commercially available high conductivity PEDOT:PSS Baytron PH500 (conductivity $~ 500 \mathrm{~S} / \mathrm{cm}$ ) as the TCE in small molecular OLED devices and achieved comparable or even superior performance (on green and blue emitting devices) than ITO anode devices [40]. Various treatments, like using zwitterionic surfactants, co-solvent system or exposing the films to dichloroacetic acid, were reported to achieve better conductivity in recent years [41-43]. Kim et al. demonstrated that with the addition of EG and a solvent post-treatment method, they could remarkably increase the conductivity up to $1418 \mathrm{~S} / \mathrm{cm}$, around $65 \mathrm{ohm} / \mathrm{sq}$ at $80 \%$ transmission [44]. Vosgueritchian et al. reported using a combination of DMSO and the fluorosurfactant Zonyl-FS300 (Zonyl) to achieve $46 \mathrm{ohm} / \mathrm{sq}$ at $82 \%$ transmission [45]. Recently, Xia et al. reported a $\mathrm{H}_{2} \mathrm{SO}_{4}$ treatment to reach $2400 \mathrm{~S} / \mathrm{cm}$ and $3065 \mathrm{~S} / \mathrm{cm}$ from multiple treatments [46]. These values are close to that of commercially available ITO substrates.

Figure 4. (a) Transmittance and sheet resistance of different polyaniline (PANI) thicknesses [34]; (b) the wavelength dependence of the refractive index and the extinction coefficient of ITO (squares) and PANI (triangles) films [35]; (c) the device structure of ITO-free transparent OLEDs based on a PEDOT:PSS TCE; (d) the comparison of external quantum efficiencies (EQEs) and corresponding photon fluxes for bottom and top emission and the sum of both (experiment (symbols) and optical simulation (lines) results); (e) aging characteristics of PEDOT:PSS-based OLEDs with a different PEDOT:PSS thickness. The encapsulated devices are aged over around 900 hours with an initial luminance of around $1500 \mathrm{~cd} / \mathrm{m}^{2}$. Constant currents are applied for each sample according to the corresponding luminance [47]. HTL, hole transport layer.

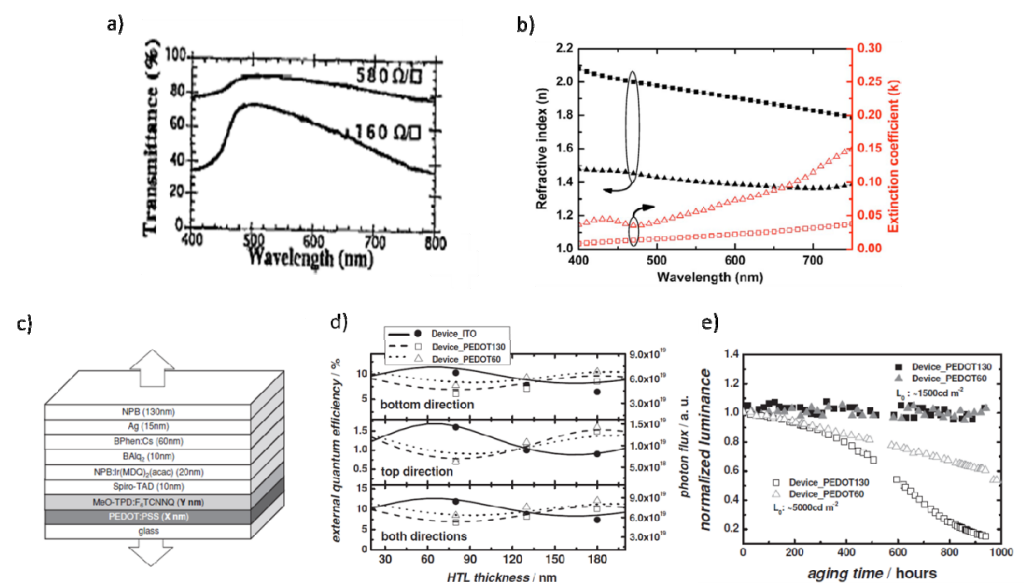

Reproduced with permission from Yang et al., Apply Physics Letter, published by the American Institute of Physics, 1994 [34]; Fehse et al., Journal of Apply Physics, published by the American Institute of Physics, 2007 [35]; and Kim et al. Advanced Materials, published by John Wiley \& Sons, Inc., 2013 [47]. 
Kim et al. demonstrated long lifetime (over $900 \mathrm{~h}$ under $1500 \mathrm{~cd} / \mathrm{m}^{2}$ ) and high performance (over 12\% external quantum efficiency (EQE) under a driving current density of $10 \mathrm{~mA} / \mathrm{cm}^{2}$ ) OLEDs on polymer TCE. The results are shown in Figure 4c-d [47], comparing with those made from the ITO substrate. Moreover, from the optical study from Cai et al, the PEDOT:PSS anode could be more beneficial to the light outcoupling than ITO substrates, due to the match of the refractive index [48]. At the current stage, however, these polymers still exhibit a lower conductivity and transmission than CNTs and metallic nanowire networks. In order to achieve a better device performance compared with ITO and other new materials, the properties and stability of the conductive polymers need to be further improved before they can be mass produced as the next generation TCE.

\section{Graphene}

Graphene is another promising candidate as a TCE in OLEDs. A flexible two-dimensional sheet of $\mathrm{sp}^{2}$-hybridized carbon atoms has very high conductivity and is nearly transparent. A single layer of graphene showed a sheet resistance of $125 \mathrm{ohm} / \mathrm{sq}$ and $97.4 \%$ transmission at the $550 \mathrm{~nm}$ wavelength, which is superior to the ITO substrate and other reported TCEs (Figure 5a) [49].

Wu et al. demonstrated solution processed graphene oxide thin films fabricated using Hummers' method and the ability to further reduce them to graphene thin films [12,50]. The graphene films were used as the TCE for OLEDs with a film thickness of about $7 \mathrm{~nm}$, a sheet resistance of about $800 \mathrm{ohm} / \mathrm{sq}$ and $82 \%$ transmission at $550 \mathrm{~nm}$, which is lower than the theoretical value, due to the existence of multiple grain boundaries, lattice defects and oxidative traps formed during the fabrication process. The device performance with a solution processed graphene electrode showed a turn-on voltage of $4.5 \mathrm{~V}$ and a luminance of $300 \mathrm{~cd} / \mathrm{m}^{2}$ at $11.7 \mathrm{~V}$, which is comparable to those of ITO substrates, which had a turn-on voltage of $3.8 \mathrm{~V}$ and a luminance of $300 \mathrm{~cd} / \mathrm{m}^{2}$ at $9.9 \mathrm{~V}$ bias (Figure 5b,c). Han et al. recently reported an extremely efficient flexible OLED by introducing a work function tunable layer [51]. The mismatch of the electrical band alignment between graphene (work function $\sim 4.4 \mathrm{eV}$ ) and the hole transport layer, like $N, N^{\prime}$-Di(1-naphthyl)- $N, N^{\prime}$-diphenyl-(1,1'-biphenyl)4,4'-diamine (NPB) (work function $\sim 5.4 \mathrm{eV}$ ), needs to be reduced for efficient hole injection. The authors incorporated a self-organized gradient hole injection layer (GraHIL), which was composed of PEDOT:PSS and tetrafluoroethylene-perfluoro-3,6-dioxa-4-methyl-7-octenesulphonic acid copolymer, one of the perfluorinated ionomers (PFIs). The GraHIL provided a work function gradient throughout the hole injection layer and, in turn, improved the charge injection efficiency. Based on a graphene/GraHIL/NPB/Alq $3 / \mathrm{LiF} / \mathrm{Al}$ structure, the luminance efficiencies with doped graphene TCE as the anode $(37.2 \mathrm{~lm} / \mathrm{W}$ in fluorescent OLEDs, $102.7 \mathrm{~lm} / \mathrm{W}$ in phosphorescent OLEDs) is higher than the devices using conventional ITO as the TCE $(24.1 \mathrm{~lm} / \mathrm{W}$ in fluorescent OLEDs, $85.6 \mathrm{~lm} / \mathrm{W}$ in phosphorescent OLEDs, shown in the Figure 5d,e). Li et al. showed that white OLEDs (WOLEDs) on graphene electrode can indeed exhibit performance satisfying general lighting requirements [52]. WOLEDs on graphene with a power efficiency of $80 \mathrm{~lm} / \mathrm{W}$ at a high brightness of $3000 \mathrm{~cd} / \mathrm{m}^{2}$ are demonstrated (Figure 5f). It is also found that graphene electrodes have the advantage of light extraction over ITO. White ITO has significant light reflection at both the top and bottom interface and also has significant light trapping. In the waveguide mode, Graphene, on the other hand, is so thin that 
it is optically negligible. There is almost no light reflection and trapping in the graphene layer. Ultimately, more light can be coupled out of the graphene OLED than the ITO OLED [52].

Figure 5. (a) Transmittance of the roll-to-roll layer-by-layer transferred graphene films on quartz substrates. The inset shows the transmittance spectra of graphene films with and without $\mathrm{HNO}_{3}$ doping and the optical images for the corresponding number of transferred layers $\left(1 \times 1 \mathrm{~cm}^{2}\right)$ [49]; (b) Current density (filled symbols) and luminance (open symbols) $v s$. applied forward bias for an OLED on graphene (squares) and ITO (circles), with an OLED device structure of anode/PEDOT:PSS/NPD/Alq3/LiF/Al; (c) External quantum efficiency (EQE) (filled symbols) and luminous power efficiency (open symbols) for an OLED on graphene film (squares) and ITO glass (circles) [12]; (d) Power efficiencies of OLED devices using various graphene layers (doped with $\mathrm{HNO}_{3}$ or $\mathrm{AuCl}_{3}$ ) and ITO as the anode; (e) Photograph of a flexible fluorescent green OLED with a four-layered graphene anode (4L-G) doped with HNO3 (4L-G-HNO3) [51]; (f) Power efficiency and current efficiency of white OLED (WOLED) based on ITO and single layer graphene electrode [52].

a)

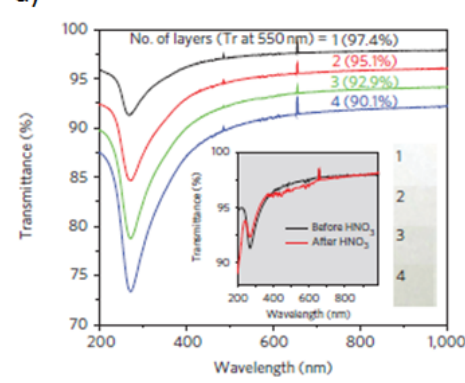

d)

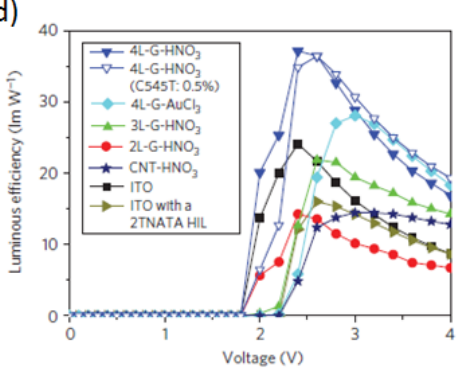

b)

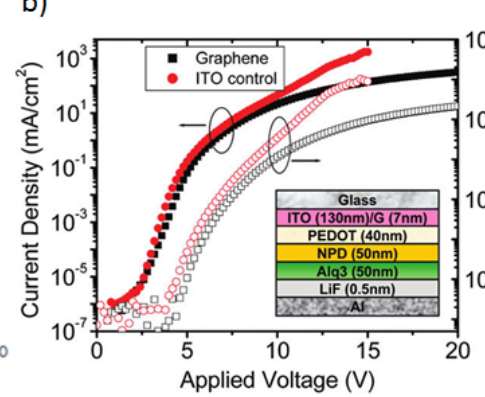

e)

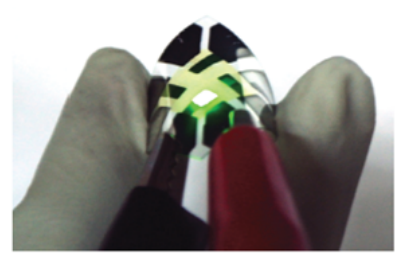

c)
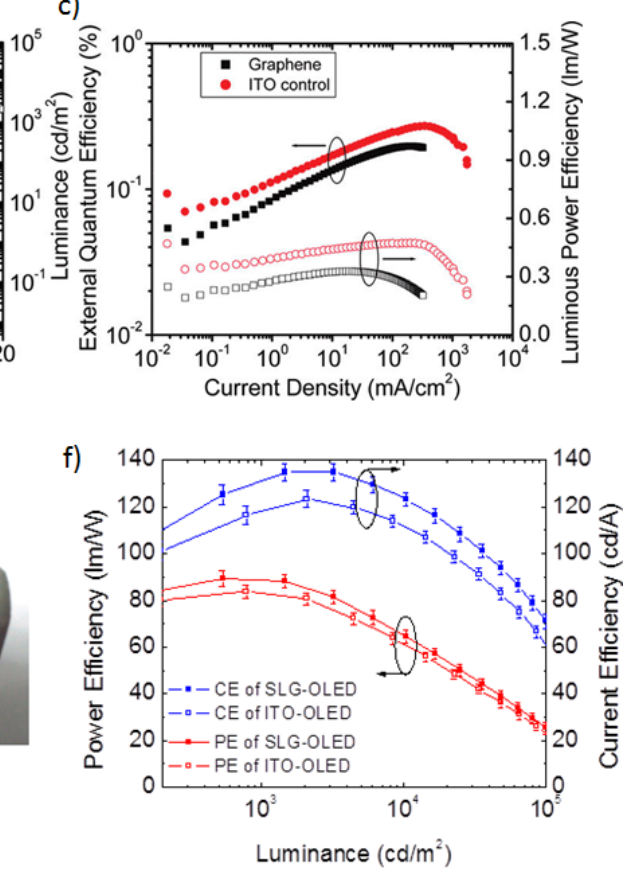

Reproduced with permission from Bae et al., Nature Nanotechnology, published by Macmillan Publishers Limited, 2010 [49]; Wu et al. ACS Nano, published by the American Chemical Society, 2010 [12]; Han et al. Nature Photonics, published by Macmillan Publishers Limited, 2012 [51]; and Li et al. Nature Communication, published by Macmillan Publishers Limited, 2013 [52].

The above high-performance OLEDs on grapheme were all demonstrated using polymer interface layers. In contrast, OLEDs on graphene only electrodes without a polymer interface layers were also reported. In the early work, Sun et al. applied multilayered graphene from chemical vapor deposition as the anode for an OLED device and showed working devices without using the polymer interface layer, but with much less performance compared with the ITO reference device. The high leakage current and low efficiency were attributed to the surface roughness and work function mismatch [53]. 
Hwang et al. found that using weak plasma treatment to multilayered graphene can improve the hole injection efficiency and demonstrated high OLED device performance on graphene without polymer interface layers [54]. These works clearly indicate graphene's capability of charge conduction and injection. After optimization, graphene can serve as an excellent electrode by itself or together with a polymer layer.

Besides the high device performance, the stability of graphene is also excellent. Due to the inert material properties, it is not reactive with most chemicals. In addition, it does not have the problem of device degradation caused by electrode material diffusion into OLEDs, which can happen for Ag and ITO electrodes. The upcoming challenge for the grapheme electrode is to further improve the electrical and optical properties and to make the fabrication process faster, easier and more cost effective.

\section{Conclusions}

Four different types of TCE materials are discussed in this review in terms of their electrical and optical performance as a TCE and the resultant OLED device performance. A comparison of their current status is summarized in Table 1. The performance of CNT TCEs is mainly limited by the contact resistance between adjacent wires, while it provides excellent mechanical properties and stability. The purity and type of CNT will also significantly affect the device performance, due to the difference in conductivity and surface roughness. Metallic nanowire TCEs exhibit better sheet resistance and transmission compared to their CNT counterparts, which resulted in highly efficient and stretchable OLED devices. Optical enhancement by the metallic nanowires is another major advantage for their future application and needs further investigation. Better control of the dimension and alignment of the metallic nanowires also needs to be further investigated. Conducting polymers have a longer developing history comparing with the other mentioned techniques. Their properties are becoming more and more competitive with traditional ITO glass showing similar conductivity values. They can also be tuned to provide optimal refractive index matching for efficient light outcoupling in OLEDs. The search for methods to precisely control the morphologies of the conducting polymers and to further improve the electrical and optical properties is ongoing. Graphene is the latest material to be applied as the TCE for OLEDs. The high transparency and the absence of light trapping from the ultrathin graphene sheet, the high in-plane conductivity and its ability to be solution processed make it a promising candidate as the next generation TCE. Its potential as the TCE in OLEDs has been proven.Outstanding OLED performance comparable to those on ITO glass has been achieved. Surface modification, the reduction of grain boundaries and defects for roll-to-roll processed graphene are some of the key routes towards further improvement.

Although most of these new TCEs still have many challenges in order to replace ITO completely, their performance is improving very rapidly. Even in the current stage, very low sheet resistance can be obtained by combining the new TCEs with a metal grid for large area applications [55]. Light extraction is an aspect that new TCEs can improve over ITO. The light reflection and trapping in most new TCEs are reduced comparing with ITO, due to the porous nature and scattering effect of CNTs and metal nanowires, the tunable index of the polymer, and the ultra-thin thickness of graphene, respectively. Because of this, light-extraction methods can be much simplified, and more light can be coupled out ultimately. In addition, new TCEs do enable many new attractive features, such as high 
mechanical flexibility and stretchability. High efficiency or reasonably good efficiency devices are demonstrated for most of these TCEs, indicating that the process integration of new TCEs into high performance OLED devices will not be a fundamental hurdle for adopting these new materials. Low cost, high throughput and reliable process development is the key to commercially viability. Once a successful process is established, the impact of a new TCE is not only limited to the field of OLED devices, but can be readily applied to various other opto electronic devices. A myriad of applications can result from the development of one alternative material. These materials and related techniques are very likely to have a significant impact on optoelectronic research and the industry in the next few decades.

Table 1. Comparison of the TCE materials.

\begin{tabular}{|c|c|c|c|c|c|}
\hline $\begin{array}{c}\text { TCE materials for } \\
\text { OLEDs }\end{array}$ & ITO & CNTs & Metal Nanowires & $\begin{array}{c}\text { Conductive } \\
\text { Polymers }\end{array}$ & Graphene \\
\hline $\begin{array}{l}\text { Conductivity/ } \\
\text { Transparency } \\
\text { (at } 550 \mathrm{~nm} \text { ) }\end{array}$ & $\begin{array}{c}10 \Omega / \mathrm{sq} \text { at } \\
90 \%\end{array}$ & $180 \Omega / \mathrm{sq}$ at $85 \%$ & $\begin{array}{c}9.7 \Omega / \mathrm{sq} \text { at } 89 \% 30 \\
\Omega / \mathrm{sq} \text { at } 93 \%\end{array}$ & $\begin{array}{l}42 \Omega / \text { sq at } 82 \% \\
240 \Omega / \text { sq at } 97 \%\end{array}$ & $\begin{array}{c}125 \Omega / \text { sq at } 97 \% \\
30 \Omega / \text { sq at } 90 \%\end{array}$ \\
\hline Light Reflection & High & Low & Low & Low & No reflection \\
\hline Light Trapping & High & Low & Low & Low & No Trapping \\
\hline Material Cost & High & Low & Medium & Low & Low \\
\hline Process Cost & High & Potentially Low & Potentially Low & Low & Potentially Low \\
\hline Stability & Good & Excellent & Medium & Medium & Excellent \\
\hline Flexibility & Poor & Flexible & Flexible & Highly flexible & Highly flexible \\
\hline $\begin{array}{c}\text { Key OLED } \\
\text { Performance } \\
\text { Demonstrated }\end{array}$ & $\begin{array}{l}>100 \mathrm{~lm} / \mathrm{W} \\
\text { (white) }\end{array}$ & $\begin{array}{l}10 \mathrm{~cd} / \mathrm{A} \text { at } \\
1000 \mathrm{~cd} / \mathrm{m} \\
(\text { green })\end{array}$ & $\begin{array}{l}54 \mathrm{~lm} / \mathrm{W} \text { similar to } \\
\text { ITO control device } \\
\text { (white) }\end{array}$ & $\begin{array}{c}12 \% \text { EQE } \\
900 \text { hours } \\
\text { (green) }\end{array}$ & $\begin{array}{c}103 \mathrm{~lm} / \mathrm{W} \text { (green) } \\
80 \mathrm{~lm} / \mathrm{W} \text { at } \\
3000 \mathrm{~cd} / \mathrm{m}^{2} \text { (white) }\end{array}$ \\
\hline Advantages over ITO & & $\begin{array}{c}\text { Solution process } \\
\text { Flexible } \\
\text { Stretchable }\end{array}$ & $\begin{array}{l}\text { Solution process } \\
\text { Flexible } \\
\text { Stretchable } \\
\text { Angle uniformity }\end{array}$ & $\begin{array}{l}\text { Solution process } \\
\text { Flexible } \\
\text { Light extraction }\end{array}$ & $\begin{array}{l}\text { Highly flexible } \\
\text { Ultra-thin } \\
\text { Light extraction }\end{array}$ \\
\hline $\begin{array}{l}\text { Challenges to } \\
\text { Replace ITO }\end{array}$ & & $\begin{array}{l}\text { Conductivity } \\
\text { Roughness } \\
\text { Cost }\end{array}$ & $\begin{array}{l}\text { Stability } \\
\text { Cost }\end{array}$ & $\begin{array}{l}\text { Conductivity } \\
\text { Stability }\end{array}$ & $\begin{array}{l}\text { Conductivity } \\
\text { Cost }\end{array}$ \\
\hline Reference & {$[56]$} & {$[23,24,57,58]$} & {$[29,30,59,60]$} & {$[45-48]$} & {$[12,49,61]$} \\
\hline
\end{tabular}

\section{Acknowledgments}

The authors would like to thank Xiaodan Zhu for proof reading.

\section{Conflicts of Interest}

The authors declare no conflict of interest.

\section{References}

1. Tsutsui, T.; Takada, N. Progress in emission efficiency of organic light-emitting diodes: Basic understanding and its technical application. Jap. J. Appl. Phys. 2013, 52, 110001. 
2. Grimsdale, A.C.; Leok Chan, K.; Martin, R.E.; Jokisz, P.G.; Holmes, A.B. Synthesis of light-emitting conjugated polymers for applications in electroluminescent devices. Chem. Rev. 2009, 109, 897-1091.

3. Li, J.; Liu, D. Dendrimers for organic light-emitting diodes. J. Mater. Chem. 2009, 19, 7584-7591.

4. Rehmann, N.; Hertel, D.; Meerholz, K.; Becker, H.; Heun, S. Highly efficient solution-processed phosphorescent multilayer organic light-emitting diodes based on small-molecule hosts. Appl. Phys. Lett. 2007, 91, 103507.

5. Gustafsson, G.; Cao, Y.; Treacy, G.M.; Klavetter, F.; Colaneri, N.; Heeger, A.J. Flexible light-emitting diodes made from soluble conducting polymers. Nature 1992, 357, 477-479.

6. Islam, A.; Rabbani, M.; Bappy, M.H.; Miah, M.A.R.; Sakib, N. A Review on Fabrication Process of Organic Light Emitting Diodes. ICIEV 2013, doi:10.1109/ICIEV.2013.6572656.

7. Tang, C.W.; VanSlyke, S.A. Organic electroluminescent diodes. Appl. Phys. Lett. 1987, 51, 913-915.

8. Gordon, R.G. Criteria for choosing transparent conductors. MRS Bull. 2000, 25, 52-57.

9. Hellstrom, S.L.; Ugolotti, J.; Britovsek, G.J.P.; Jones, T.S.; White, A.J.P. The effect of fluorination on the luminescent behaviour of 8-hydroxyquinoline boron compounds. New J. Chem. 2008, 32, 1379-1387.

10. Peng, C.; Jia, Z.; Neilson, H.; Li, T.; Lou, J. In situ electro-mechanical experiments and mechanics modeling of fracture in indium tin oxide-based multilayer electrodes. Adv. Eng. Mater. 2012, 15, 250-256.

11. Kim, N.-R.; Lee, J.-H.; Lee, Y.-Y.; Nam, D.-H.; Yeon, H.-W.; Lee, S.-Y.; Yang, T.-Y.; Lee, Y.-J.; Chu, A.; Nam, K.T.; et al. Enhanced conductivity of solution-processed indium tin oxide nanoparticle films by oxygen partial pressure controlled annealing. J. Mater. Chem. C 2013, 1, 5953-5959.

12. Wu, J.; Agrawal, M.; Becerril, H.C.A.; Bao, Z.; Liu, Z.; Chen, Y.; Peumans, P. Organic light-emitting diodes on solution-processed graphene transparent electrodes. ACS Nano 2009, 4, 43-48.

13. Zhang, M.; Fang, S.; Zakhidov, A.A.; Lee, S.B.; Aliev, A.E.; Williams, C.D.; Atkinson, K.R.; Baughman, R.H. Strong, transparent, multifunctional, carbon nanotube sheets. Science 2005, 309, 1215-1219.

14. Hu, L.; Hecht, D.S.; Gruner, G. Carbon nanotube thin films: Fabrication, properties, and applications. Chem. Rev. 2010, 110, 5790-5844.

15. De Volder, M.F.L.; Tawfick, S.H.; Baughman, R.H.; Hart, A.J. Carbon nanotubes: Present and future commercial applications. Science 2013, 339, 535-539.

16. Dresselhaus, M.; Dresselhaus, G.; Avouris, P.; Ajayan, P.; Zhou, O. Applications of Carbon Nanotubes. In Carbon Nanotubes; Springer: Berlin/Heidelberg, Germany, 2001; Volume 80, pp. 391-425.

17. Martel, R.; Schmidt, T.; Shea, H.R.; Hertel, T.; Avouris, P. Single- and multi-wall carbon nanotube field-effect transistors. Appl. Phys. Lett. 1998, 73, 2447-2449.

18. Zhao, J.; Han, J.; Lu, J.P. Work functions of pristine and alkali-metal intercalated carbon nanotubes and bundles. Phys. Rev. B 2002, 65, 193401.

19. Suzuki, S.; Bower, C.; Watanabe, Y.; Zhou, O. Work functions and valence band states of pristine and cs-intercalated single-walled carbon nanotube bundles. Appl. Phys. Lett. 2000, 76, 4007-4009. 
20. Zhang, D.; Ryu, K.; Liu, X.; Polikarpov, E.; Ly, J.; Tompson, M.E.; Zhou, C. Transparent, conductive, and flexible carbon nanotube films and their application in organic light-emitting diodes. Nano Lett. 2006, 6, 1880-1886.

21. Li, J.; Hu, L.; Wang, L.; Zhou, Y.; Gruner, G.; Marks, T.J. Organic light-emitting diodes having carbon nanotube anodes. Nano Lett. 2006, 6, 2472-2477.

22. Ou, E.C.W.; Hu, L.; Raymond, G.C.R.; Soo, O.K.; Pan, J.; Zheng, Z.; Park, Y.; Hecht, D.; Irvin, G.; Drzaic, P.; et al. Surface-modified nanotube anodes for high performance organic light-emitting diode. ACS Nano 2009, 3, 2258-2264.

23. Yu, Z.; Hu, L.; Liu, Z.; Sun, M.; Wang, M.; Gruner, G.; Pei, Q. Fully bendable polymer light emitting devices with carbon nanotubes as cathode and anode. Appl. Phys. Lett. 2009, 95, 203304.

24. Yu, Z.; Niu, X.; Liu, Z.; Pei, Q. Intrinsically stretchable polymer light-emitting devices using carbon nanotube-polymer composite electrodes. Adv. Mater. 2011, 23, 3989-3994.

25. Hu, L.; Wu, H.; Cui, Y. Metal nanogrids, nanowires, and nanofibers for transparent electrodes. MRS Bull. 2011, 36, 760-765.

26. Lee, J.-Y.; Connor, S.T.; Cui, Y.; Peumans, P. Solution-processed metal nanowire mesh transparent electrodes. Nano Lett. 2008, 8, 689-692.

27. Yu, Z.; Zhang, Q.; Li, L.; Chen, Q.; Niu, X.; Liu, J.; Pei, Q. Highly flexible silver nanowire electrodes for shape-memory polymer light-emitting diodes. Adv. Mater. 2011, 23, 664-668.

28. Gaynor, W.; Hofmann, S.; Christoforo, M.G.; Sachse, C.; Mehra, S.; Salleo, A.; McGehee, M.D.; Gather, M.C.; Lüssem, B.; Müller-Meskamp, L.; et al. Color in the corners: ITO-free white oleds with angular color stability. Adv. Mater. 2013, 25, 4006-4013.

29. Liang, J.; Li, L.; Niu, X.; Yu, Z.; Pei, Q. Elastomeric polymer light-emitting devices and displays. Nat. Photon. 2013, 7, 817-824.

30. Song, T.-B.; Chen, Y.; Chung, C.-H.; Yang, Y.; Bob, B.; Duan, H.-S.; Li, G.; Tu, K.-N.; Huang, Y. Nanoscale joule heating and electromigration enhanced ripening of silver nanowire contacts. ACS Nano ASAP 2014, doi:10.1021/nn4065567.

31. Karim, S.; Toimil-Molares, M.E.; Balogh, A.G.; Ensinger, W.; Cornelius, T.W.; Khan, E.U.; Neumann, R. Morphological evolution of au nanowires controlled by rayleigh instability. Nanotechnology 2006, 17, 5954-5959.

32. Hsu, S.-Y.; Lee, M.-C.; Lee, K.-L.; Wei, P.-K. Extraction enhancement in organic light emitting devices by using metallic nanowire arrays. Appl. Phys. Lett. 2008, 92, 013303.

33. Cao, Y.; Treacy, G.M.; Smith, P.; Heeger, A.J. Solutioncast films of polyaniline: Optical quality transparent electrodes. Appl. Phys. Lett. 1992, 60, 2711-2713.

34. Yang, Y.; Heeger, A.J. Polyaniline as a transparent electrode for polymer lightemitting diodes: Lower operating voltage and higher efficiency. Appl. Phys. Lett. 1994, 64, 1245-1247.

35. Fehse, K.; Schwartz, G.; Walzer, K.; Leo, K. Combination of a polyaniline anode and doped charge transport layers for high-efficiency organic light emitting diodes. J. Appl. Phys. 2007, 101, 124509.

36. Bayer, A.G. Eur. Patent, 440957, 1991.

37. Cao, Y.; Yu, G.; Zhang, C.; Menon, R.; Heeger, A.J. Polymer light-emitting diodes with polyethylene dioxythiophene-polystyrene sulfonate as the transparent anode. Synth. Met. 1997, 87, 171-174. 
38. Kim, J.Y.; Jung, J.H.; Lee, D.E.; Joo, J. Enhancement of electrical conductivity of poly(3,4ethylenedioxythiophene)/poly(4-styrenesulfonate) by a change of solvents. Synth. Met. 2002, 126, 311-316.

39. Ouyang, J.; Chu, C.W.; Chen, F.C.; Xu, Q.; Yang, Y. High-conductivity poly(3,4ethylenedioxythiophene):Poly(styrene sulfonate) film and its application in polymer optoelectronic devices. Adv. Funct. Mater. 2005, 15, 203-208.

40. Fehse, K.; Walzer, K.; Leo, K.; Lövenich, W.; Elschner, A. Highly conductive polymer anodes as replacements for inorganic materials in high-efficiency organic light-emitting diodes. Adv. Mater. 2007, 19, 441-444.

41. Xia, Y.; Zhang, H.; Ouyang, J. Highly conductive PEDOT:PSS films prepared through a treatment with zwitterions and their application in polymer photovoltaic cells. J. Mater. Chem. 2010, 20, 9740-9747.

42. Xia, Y.; Ouyang, J. PEDOT:PSS films with significantly enhanced conductivities induced by preferential solvation with cosolvents and their application in polymer photovoltaic cells. J. Mater. Chem. 2011, 21, 4927-4936.

43. Yoo, J.E.; Lee, K.S.; Garcia, A.; Tarver, J.; Gomez, E.D.; Baldwin, K.; Sun, Y.; Meng, H.; Nguyen, T.-Q.; Loo, Y.-L. Directly patternable, highly conducting polymers for broad applications in organic electronics. Proc. Natl. Acad. Sci. USA 2010, 107, 5712-5717.

44. Kim, Y.H.; Sachse, C.; Machala, M.L.; May, C.; Müller-Meskamp, L.; Leo, K. Highly conductive PEDOT:PSS electrode with optimized solvent and thermal post-treatment for ito-free organic solar cells. Adv. Funct. Mater. 2011, 21, 1076-1081.

45. Vosgueritchian, M.; Lipomi, D.J.; Bao, Z. Highly conductive and transparent PEDOT:PSS films with a fluorosurfactant for stretchable and flexible transparent electrodes. Adv. Funct. Mater. 2012, 22, 421-428.

46. Xia, Y.; Sun, K.; Ouyang, J. Solution-processed metallic conducting polymer films as transparent electrode of optoelectronic devices. Adv. Mater. 2012, 24, 2436-2440.

47. Kim, Y.H.; Lee, J.; Hofmann, S.; Gather, M.C.; Müller-Meskamp, L.; Leo, K. Achieving high efficiency and improved stability in ito-free transparent organic light-emitting diodes with conductive polymer electrodes. Adv. Funct. Mater. 2013, 23, 3763-3769.

48. Cai, M.; Ye, Z.; Xiao, T.; Liu, R.; Chen, Y.; Mayer, R.W.; Biswas, R.; Ho, K.-M.; Shinar, R.; Shinar, J. Extremely efficient indium-tin-oxide-free green phosphorescent organic light-emitting diodes. Adv. Mater. 2012, 24, 4337-4342.

49. Bae, S.; Kim, H.; Lee, Y.; Xu, X.; Park, J.-S.; Zheng, Y.; Balakrishnan, J.; Lei, T.; Kim, H.R.; Song, Y.I.; et al. Roll-to-roll production of 30-inch graphene films for transparent electrodes. Nat. Nanotechnol. 2010, 5, 574-578.

50. Hummers, W.S.; Offeman, R.E. Preparation of graphitic oxide. J. Am. Chem. Soc. 1958, 80, 1339-1339.

51. Han, T.-H.; Lee, Y.; Choi, M.-R.; Woo, S.-H.; Bae, S.-H.; Hong, B.H.; Ahn, J.-H.; Lee, T.-W. Extremely efficient flexible organic light-emitting diodes with modified graphene anode. Nat. Photon. 2012, 6, 105-110. 
52. Li, N.; Oida, S.; Tulevski, G.S.; Han, S.-J.; Hannon, J.B.; Sadana, D.K.; Chen, T.-C. Efficient and bright organic light-emitting diodes on single-layer graphene electrodes. Nat. Commun. 2013, 4, 2294.

53. Sun, T.; Wang, Z.L.; Shi, Z.J.; Ran, G.Z.; Xu, W.J.; Wang, Z.Y.; Li, Y.Z.; Dai, L.; Qin, G.G. Multilayered graphene used as anode of organic light emitting devices. Appl. Phys. Lett. 2010, 96, 133301.

54. Hwang, J.; Choi, H.K.; Moon, J.; Kim, T.Y.; Shin, J.-W.; Joo, C.W.; Han, J.-H.; Cho, D.-H.; Huh, J.W.; Choi, S.-Y.; et al. Multilayered graphene anode for blue phosphorescent organic light emitting diodes. Appl. Phys. Lett. 2012, 100, 133304.

55. Zhu, Y.; Sun, Z.; Yan, Z.; Jin, Z.; Tour, J.M. Rational design of hybrid graphene films for high-performance transparent electrodes. ACS Nano 2011, 5, 6472-6479.

56. Ellmer, K. Past achievements and future challenges in the development of optically transparent electrodes. Nat. Photon. 2012, 6, 809-817.

57. Nirmalraj, P.N.; Lyons, P.E.; De, S.; Coleman, J.N.; Boland, J.J. Electrical connectivity in single-walled carbon nanotube networks. Nano Lett. 2009, 9, 3890-3895.

58. Gruner, G. Carbon nanotube films for transparent and plastic electronics. J. Mater. Chem. 2006, 16, 3533-3539.

59. Chung, C.-H.; Song, T.-B.; Bob, B.; Zhu, R.; Duan, H.-S.; Yang, Y. Silver Nanowire Composite Window Layers for Fully Solution-Deposited Thin-Film Photovoltaic Devices. Adv. Mater. 2012, 24, 5499-5504.

60. Leem, D.-S.; Edwards, A.; Faist, M.; Nelson, J.; Bradley, D.D.C.; De Mello, J.C. Efficient organic solar cells with solution-processed silver nanowire electrodes. Adv. Mater. 2011, 23, 4371-4375.

61. Eda, G.; Fanchini, G.; Chhowalla, M. Large-area ultrathin films of reduced graphene oxide as a transparent and flexible electronic material. Nat. Nanotechnol. 2008, 3, 270-274.

(C) 2014 by the authors; licensee MDPI, Basel, Switzerland. This article is an open access article distributed under the terms and conditions of the Creative Commons Attribution license (http://creativecommons.org/licenses/by/3.0/). 